\title{
Nona-Arginine Facilitates Delivery of Quantum Dots into Cells via Multiple Pathways
}

\author{
Yi Xu, ${ }^{1}$ Betty Revon Liu, ${ }^{2}$ Han-Jung Lee, ${ }^{2}$ Katie B. Shannon, ${ }^{1}$ Jeffrey G. Winiarz, ${ }^{3}$ \\ Tien-Chun Wang, ${ }^{4}$ Huey-Jenn Chiang, ${ }^{5}$ and Yue-wern Huang ${ }^{1}$ \\ ${ }^{1}$ Department of Biological Sciences, Missouri University of Science and Technology, 105 Schrenk Hall, 400 W. 11th Street, Rolla, \\ MO 65409, USA \\ ${ }^{2}$ Department of Natural Resources and Environmental Studies and Institute of Biotechnology, National Dong Hwa University, \\ Hualien 97401, Taiwan \\ ${ }^{3}$ Department of Chemistry, Missouri University of Science and Technology, 142 Schrenk Hall, 400 W. 11th Street, Rolla, \\ MO 65409, USA \\ ${ }^{4}$ Department of Life Science, National Taiwan Normal University, 88 Ting-Chow Road, Section 4, Taipei 116, Taiwan \\ ${ }^{5}$ Institute of Biotechnology, National Dong Hwa University, Hualien 97401, Taiwan
}

Correspondence should be addressed to Han-Jung Lee, hjlee@mail.ndhu.edu.tw and Yue-wern Huang, huangy@mst.edu

Received 26 April 2010; Revised 3 August 2010; Accepted 26 September 2010

Academic Editor: Lokesh Joshi

Copyright ( 92010 Yi Xu et al. This is an open access article distributed under the Creative Commons Attribution License, which permits unrestricted use, distribution, and reproduction in any medium, provided the original work is properly cited.

Semiconductor quantum dots (QDs) have recently been used to deliver and monitor biomolecules, such as drugs and proteins. However, QDs alone have a low efficiency of transport across the plasma membrane. In order to increase the efficiency, we used synthetic nona-arginine (SR9), a cell-penetrating peptide, to facilitate uptake. We found that SR9 increased the cellular uptake of QDs in a noncovalent binding manner between QDs and SR9. Further, we investigated mechanisms of QD/SR9 cellular internalization. Low temperature and metabolic inhibitors markedly inhibited the uptake of QD/SR9, indicating that internalization is an energy-dependent process. Results from both the pathway inhibitors and the RNA interference (RNAi) technique suggest that cellular uptake of QD/SR9 is predominantly a lipid raft-dependent process mediated by macropinocytosis. However, involvement of clathrin and caveolin-1 proteins in transducing QD/SR9 across the membrane cannot be completely ruled out.

\section{Introduction}

Fluorescent semiconductor quantum dots (QDs) have been used to deliver and monitor biomolecules into cells in the past few years [1-3]. Advantages of QDs over traditional dyes and proteins (e.g., green and red fluorescent proteins) include their unique physical and chemical properties, namely, photostability, high quantum yield, narrow emission peak, exceptional resistance to degradation, broad sizedependent photoluminescence, and multiplexing potential [4]. Although QDs can be engulfed by living cells, the use of cell-penetrating peptides can increase uptake efficiency [5].

The study of the uptake mechanism of QDs is just beginning. Using specific inhibitors, Ruan et al. identified macropinocytosis, actin filaments, and microtubules as required for internalization and intracellular transport of streptavidin-coated QD/Tat-biotin in HeLa cells [6]. Zhang and Monteiro-Riviere demonstrated that carboxylic QDs were internalized by lipid raft-dependent endocytosis in human epidermal keratinocytes, and these pathways were primarily regulated by the G-protein-coupled receptor associated pathway and low-density lipoprotein receptor/scavenger receptor [7].

Cell-penetrating peptides (CPPs), also known as protein transduction domains (PTDs) or membrane transduction peptides (MTPs), have been used to transduce biologically active proteins, siRNA, and drugs across plasma membranes $[8,9]$. The advantages of CPPs include ease of preparation, lack of toxicity to the cell, and high efficiency of 
transduction [10]. CPPs can enter cells with a half-time of 1.8 minutes, corresponding to a first-order rate constant $\mathrm{k}$ of $0.007 \mathrm{sec}^{-1}$ [11]. Among the basic CPPs, the cellular uptake of polyarginine tends to be more efficient than that of polylysine, polyhistidine, or polyornithine [12]. The highest translocation efficiencies were achieved by using octaarginine or nona-arginine peptides [12]. The synthetic nonaarginine (SR9) peptide has been shown to effectively deliver not only covalently fused proteins but also noncovalently bound protein into different types of animal and plant cells $[9,13]$.

The mechanism of cellular entry of CPPs has been the focus of numerous studies. Early reports suggested that CPPs delivery of molecules into cells was independent of endocytosis, energy, receptors, or active transporters [1416]. However, it was later found that fixing cells may have artificially transduced molecules across plasma membranes [17]. More recent studies using live cell imaging suggested the involvement of macropinocytosis $[18,19]$.

The goals of this study were to determine (1) whether SR9 can noncovalently facilitate QD uptake and (2) the internalization mechanisms for uptake of QD/SR9 complex. We treated A549 cells with QDs alone or QD/SR9 complex to determine the efficiency of QD/SR9 uptake. Inhibitors and siRNA were used to identify molecules and processes that contribute to the uptake.

\section{Materials and Methods}

2.1. Quantum Dots. CdSe/ZnS quantum dots (Adirondack Green, $520 \mathrm{~nm}$ ) were purchased from Evident Technologies (Troy, NK, USA). These quantum dots have a polyethylene glycol (PEG) lipid surface coated with carboxyl terminal groups. The emission and excitation peak wavelengths are $520 \mathrm{~nm}$ and $505 \mathrm{~nm}$, respectively. The hydrodynamic diameter is $25 \mathrm{~nm}$.

2.2. Nona-Arginines (SR9). Nona-arginines were synthesized by solid-phase peptide synthesis (Sigma-Aldrich, Saint Louis, MO, USA). The synthetic peptides were purified by high performance liquid chromatography (HPLC) using a reverse phase column. The purity of SR9 was $\sim 99 \%$.

2.3. Chemicals. Fetal bovine serum, Ham's F-12 medium with L-glutamine, trypsin-EDTA (1x), penicillinstreptomycin, sucrose, sodium azide, sodium fluoride, and chlorpromazine were purchased from Fisher Scientific (Pittsburgh, PA, USA). Nystatin, filipin, 5-(N-ethyl-Nisopropyl) amiloride (EIPA), cytochalasin D (Cyt D), antimycin A, and monodansylcadaverine (MDC) were purchased from Sigma-Aldrich.

2.4. Cell Culture. The human bronchoalveolar carcinomaderived cell line (A549) was purchased from ATCC (Manassas, VA, USA). Cells were maintained in Ham's F-12 medium supplemented with $10 \%$ fetal bovine serum, 100 units $/ \mathrm{mL}$ penicillin, and $100 \mu \mathrm{g} / \mathrm{mL}$ streptomycin and grown at $37^{\circ} \mathrm{C}$ in a 5\% $\mathrm{CO}_{2}$ humidified environment. A549 cells were seeded

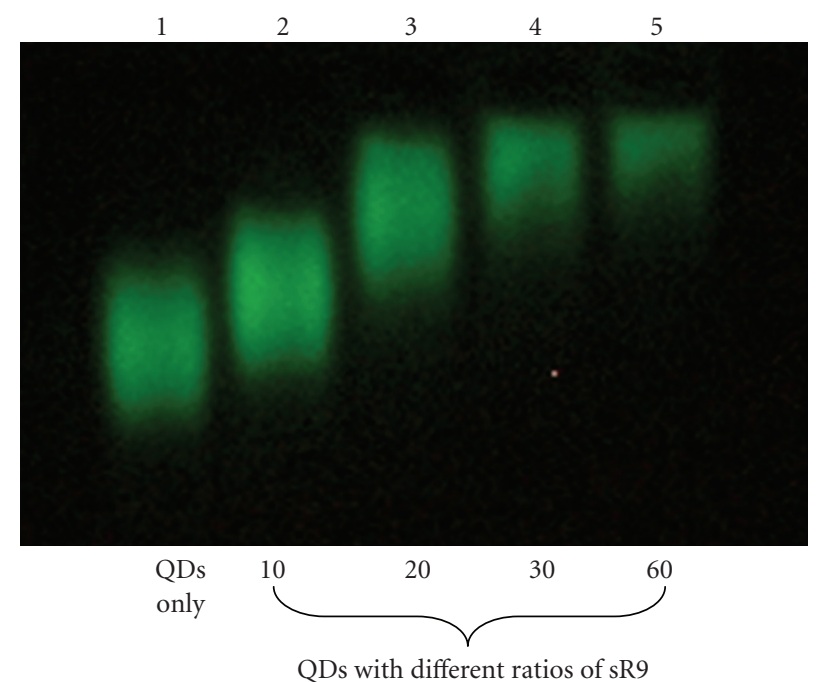

FIGURE 1: Gel retardation analysis of the interaction between QDs and SR9. QDs were premixed with SR9 at different molecular ratios. Lane 1: QDs without SR9. Lanes 2-5: QDs mixed with SR9 at ratios of $10,20,30$, and 60 , respectively. The decrease in mobility reflected the formation of QD/SR9 complexes.

into $35 \mathrm{~mm}$ glass-bottom tissue culture plates (MatTek, Ashland, MA, USA) at an initial confluency of $20 \%$ and allowed to attach for 48 hours.

2.5. Gel Retardation Assay. The QD and SR9 mixtures were prepared at different molecular ratios $(1: 10,1: 20,1: 30$, and $1: 60)$ and then incubated for $20 \mathrm{~min}$ at room temperature to allow noncovalent complexes to form. These complexes were analyzed by electrophoresis in a $0.6 \%$ agarose gel in $0.5 \%$ TAE buffer at 130 voltages for $60 \mathrm{~min}$. The complexes were visualized by UV light.

2.6. QD Uptake with SR9 at Different Molecular Ratios. To determine the optimal QD/SR9 ratio for efficient delivery, A549 cells were incubated with QD/SR9 at different molecular ratios ( $1: 10$ to $1: 60)$. The cells were washed with icecold phosphate buffered saline (PBS) six times to remove unbound QD/SR9. Fluorescent images were then obtained and quantified.

2.7. Cytotoxicity Measurement. To determine the cytotoxicity of QD/SR9, A549 cells were treated with QD/SR9 (1:20) at $12.5,25,50,100$, and $200 \mathrm{nM}$ QD concentrations for 24 hours. Untreated cells served as a control group. The MTS assay (Cell Titer 96 Aqueous One Solution Assay, Promega, Madison, WI, USA) was used to determine cytotoxicity. Absorbance was measured at $490 \mathrm{~nm}$ using a microplate reader (FLOUstar, BMG Labtechnologies, Durham, NC, USA).

2.8. Noncovalent Binding and Internalization of QDs and SR9. QDs were premixed with SR9 peptides at the molecular ratio of $1: 20$ at room temperature for $20 \mathrm{~min}$. immediately 


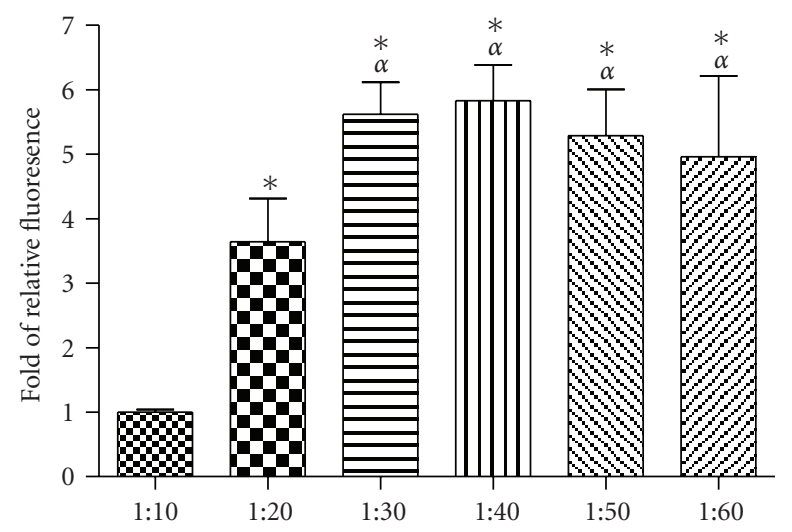

FIgURE 2: QD uptake with SR9 at different molecular ratios. QDs were premixed with SR9 at molecular ratios from $1: 10$ to $1: 60$. A549 cells were treated with QD/SR9. Values are mean \pm SD from three independent experiments. Fluorescence intensity is normalized relative to the value at $1: 10 .{ }^{*} P<.01$ versus $1: 10$; ${ }^{\alpha} P<.01$ versus $1: 20$.

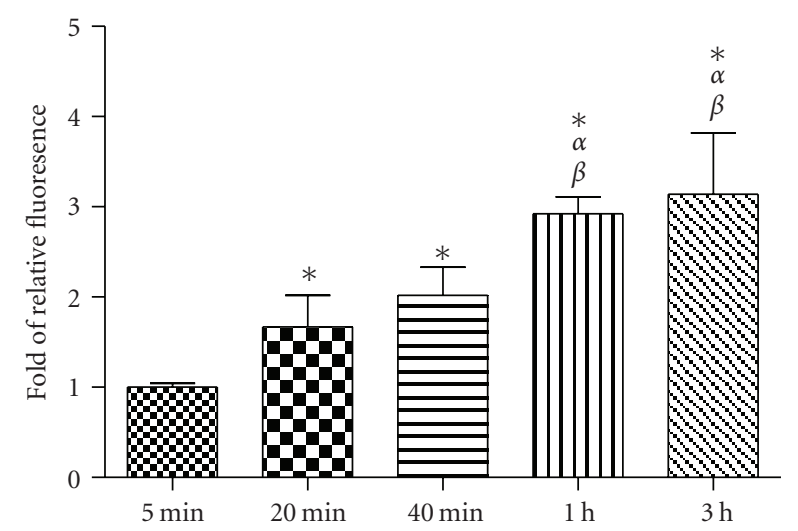

FIGURE 3: Time-dependent uptake of QD/SR9. QDs were premixed with SR9 at a molecular ratio of $1: 20$. A549 cells were treated with QD/SR9 at $150 \mathrm{nM}$ QD concentration for $5 \mathrm{~min}, 20 \mathrm{~min}, 40 \mathrm{~min}, 1$ hour, and 3 hours. Values are mean \pm SD from three independent experiments. Fluorescence intensity is normalized relative to the value at $5 \mathrm{~min} .{ }^{*} P<.01$ versus $5 \mathrm{~min} ;{ }^{\alpha} P<.01$ versus $20 \mathrm{~min}$; $\beta P<.01$ versus $40 \mathrm{~min}$.

upon removal of medium, the cells were treated with the QD/SR9 mixture at a final QD concentration of $150 \mathrm{nM}$ for a designated period of time. After treatment, cells were washed six times with ice-cold PBS. Phenol red-free medium $(2 \mathrm{~mL})$ (Invitrogen, Carlsbad, CA, USA) was added for fluorescent image studies.

\subsection{Uptake Studies}

Energy Inhibition. To determine whether uptake of QD/SR9 is energy dependent, cells were incubated with QD/SR9 under varying metabolic conditions. In temperature studies, cells were treated at either $37^{\circ} \mathrm{C}$ or $4^{\circ} \mathrm{C}$. In the low temperature group, the cells were preincubated at $4^{\circ} \mathrm{C}$ for

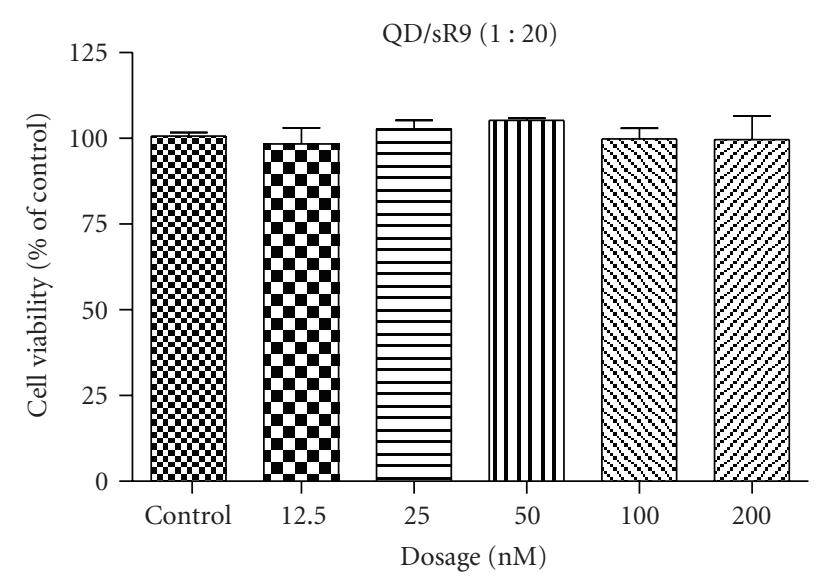

FIgure 4: Cell viability of QD/SR9-treated cells. A549 cells were treated with QD/SR9 $(1: 20)$ for 24 hours. The concentrations of QDs used were 12.5, 25, 50, 100, and $200 \mathrm{nM}$. Values are mean \pm SD from three independent experiments.

30 min and then treated with QD/SR9 at $4^{\circ} \mathrm{C}$ for another 1hour. Cells maintained at $37^{\circ} \mathrm{C}$ comprised the control group. In metabolic inhibition experiments, cells were incubated in the absence or presence of a mixture of metabolic inhibitors $(0.15 \%$ sodium azide, $15 \mathrm{mM}$ sodium fluoride, and $2 \mu \mathrm{g} / \mathrm{mL}$ antimycin A). Cells were pretreated with a mixture of metabolic inhibitors for 1 hour followed by addition of QD/SR9 to the cells and then incubated for another 1 hour.

Clathrin-Dependant Pathway Inhibition. To disrupt clathrindependent endocytosis, hypertonic challenge, chlorpromazine, and MDC were used. Cells were pretreated with $0.45 \mathrm{M}$ sucrose, $10 \mu \mathrm{M}$ chlorpromazine, or $25 \mu \mathrm{g} / \mathrm{mL} \mathrm{MDC}$ for $30 \mathrm{~min}$. QD/SR9 was then added to the cells followed by 1 hour incubation.

Caveolin-Dependant Pathway Inhibition. To inhibit caveolindependent endocytosis, filipin and nystatin were used to deplete cholesterol. Cells were preincubated with $3 \mu \mathrm{g} / \mathrm{ml}$ filipin and $20 \mu \mathrm{g} / \mathrm{ml}$ nystatin for $30 \mathrm{~min}$ before treatment with QD/SR9 for another 1 hour.

Macropinocytosis Pathway Inhibition. EIPA $(30 \mu \mathrm{M})$ or Cyt D $(1 \mu \mathrm{g} / \mathrm{mL})$ was used to block macropinocytosis. Cells were pretreated with the inhibitor for $30 \mathrm{~min}$ before treatment with QD/SR9 for another 1 hour.

2.10. Small Interfering RNA (siRNA) Transfection. Due to potential nonspecific effects and toxicity of pharmacological inhibitors, clathrin heavy chain and caveolin-1 siRNA (Sigma-Aldrich) were used to depress expression of critical components of the clathrin and caveolar pathways. The sense and antisense sequences of these siRNA were as follows.

Clathrin HC:

5' -CCCUAAACACCUCAACGAU-3' (sense)

5'-AUCGUUGAGGUGUUUAGGG-3' (antisense). 


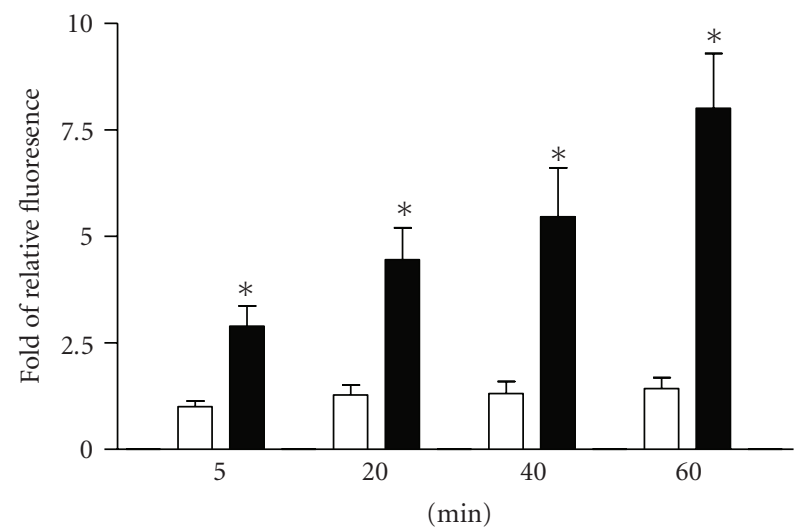

QDs

QD/SR9

(a)

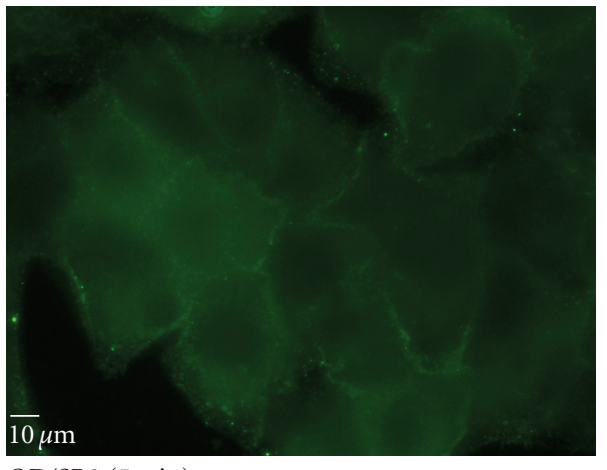

QD/SR9 (5 min)

(b)

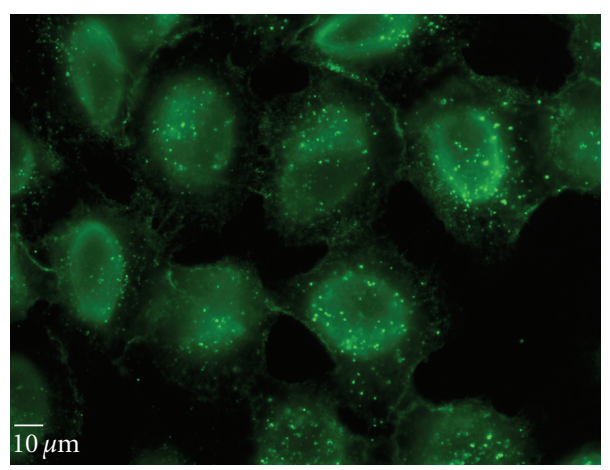

QD/SR9 (1 hour)

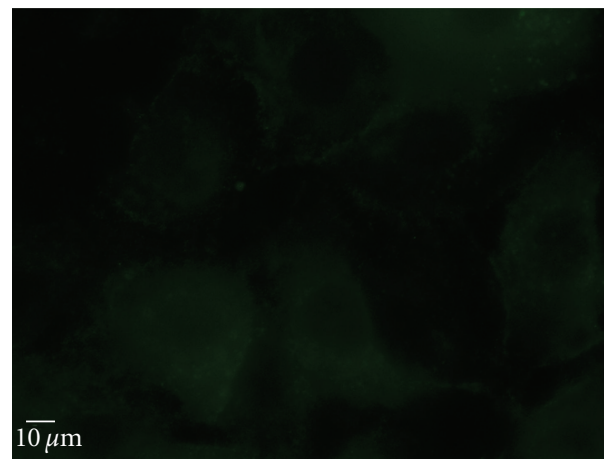

QDs (5 min)

(c)

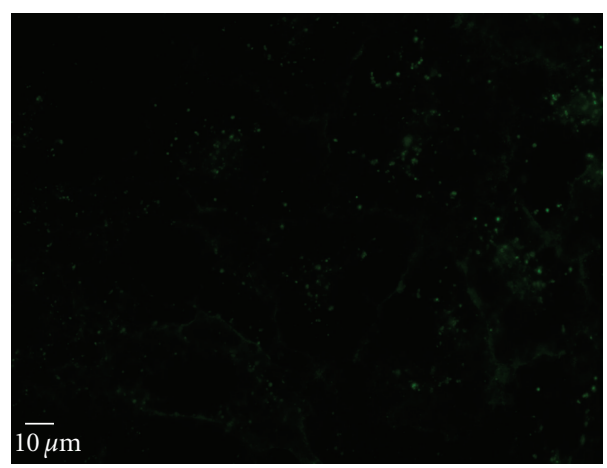

QDs (1 hour)

(d)

(e)

FIGURE 5: SR9 facilitates QD uptake. (a) The relative QD fluorescence intensity in cells exposed to QD or QD/SR9. A549 cells were treated with $150 \mathrm{nM}$ QDs and $150 \mathrm{nM}$ QD/SR9 (1:20) for $5 \mathrm{~min}, 20 \mathrm{~min}, 40 \mathrm{~min}$, and $60 \mathrm{~min}$. Fluorescence intensity is normalized relative to QDs alone at 5 min. (b) and (c) QD fluorescence in cells with and without SR9 after a 5-minute exposure. (d) and (e) QD fluorescence in cells with and without SR9 after a 1-hour exposure. Images are from a single plane of Z series. Values are mean \pm SD from three independent experiments. Significance is indicated by ${ }^{*} P<.01$ versus QDs-alone group.

Caveolin-1:

5'-CAUUAUGACCGGGCUCAUA-3' (sense)

5'-UAUGAGCCCGGUCAUAAUG-3' (antisense).
Transfection of siRNA into the A549 cells was performed at a $\sim 30 \%$ cell confluence. siRNA was complexed with Lipofectamine 2000 reagent according to the manufacturer's instructions (Invitrogen). Western blot and QD/SR9 uptake studies were conducted 3 days after transfection. 


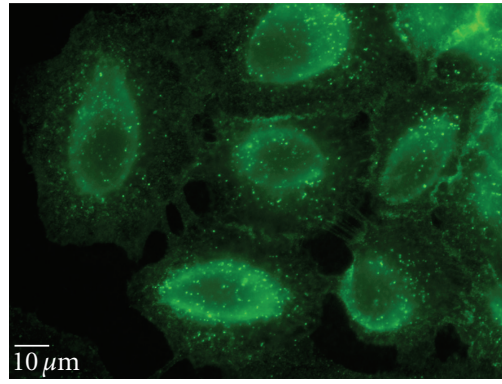

(a)

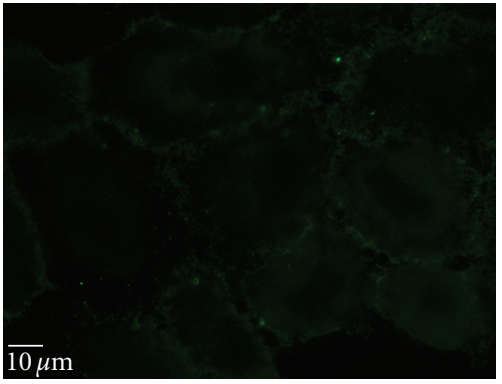

(b)

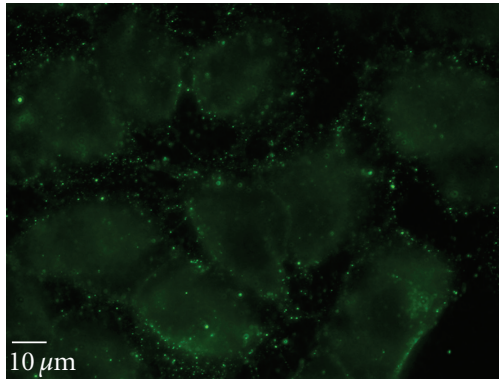

(c)

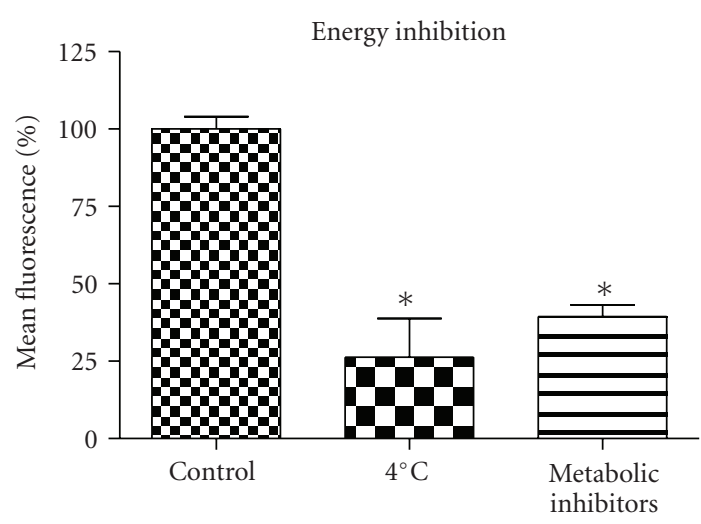

(d)

FIGURE 6: Energy inhibition reduced internalization of QD/SR9 (150 nM; 1:20; 1 hour). (a) Control cells. (b) Cells pretreated at $4{ }^{\circ} \mathrm{C}$ for 30 min then incubated with QD/SR9 for an additional 1 hour. (c) Cells pretreated with a metabolic inhibitor mixture for 1 hour then incubated with QD/SR9 for an additional 1 hour. Images are from a single plane of Z series. (d) Fluorescence intensity. Values are mean \pm SD from three independent experiments $\left({ }^{*} P^{\prime}\right.$ s $<.01$ versus control).

2.11. Western Blot Analysis. A549 cells were lysed in a buffer comprised of $150 \mathrm{mM}$ sodium chloride, $1.0 \%$ Triton X-100, and $50 \mathrm{mM}$ Tris, $\mathrm{pH} 8.0$, containing $1 \%$ protease inhibitor cocktail (Sigma-Aldrich). The protein concentration was quantified by the Bradford procedure (BioRad, Hercules, CA, USA). Ten $\mu$ g total cell protein was electrophoresed on $8 \%$ (clathrin-heavy chain) or 12\% (caveolin-1) SDS-PAGE, followed by electrotransfer to a nitrocellulose membrane. Clathrin-heavy chain was detected using a mouse monoclonal antibody at a dilution of 1:200 and goat antimouse IgG-HRP secondary antibody (Santa Cruz, CA, USA). Caveolin-1 protein was detected using a rabbit monoclonal antibody at a dilution of $1: 1000$ and goat antirabbit IgGHRP secondary antibody (Cell Signaling, Danvers, MA, USA). The blots were probed with the ECL Western blot detection system according to the manufacturer's instructions (Thermo Fisher Scientific, Rockford, IL, USA). The Image software (NIH) was used for quantitative analysis of the Western blot.

2.12. Epifluorescent Microscopy. After treatment with QD/SR9, cells were viewed with an Olympus IX51 inverted microscope at $600 \mathrm{X}$ total magnification using a UPLFLN 60X NA 1.25 objective (Olympus, Center Valley, PA, USA). QD filter set (EX 435/40, EM 519-700) was used for QDs (Semrock, Rochester, NY, USA). Images were captured with a Hamamatsu ORCA285 CCD camera. Z series images of the entire cell volume were taken at $0.3 \mu \mathrm{m}$ internals. Images were analyzed using SlideBook software (Intelligent Imaging Innovations, Denver, CO, USA).

2.13. Statistical Analyses. Data were expressed as the mean \pm standard deviation from three independent experiments. Fluorescent intensity was quantified using SlideBook software. The mean fluorescence intensity (after background subtraction) of a single plane in $\mathrm{Z}$ series was recorded. Onetailed unpaired Student's $t$-test was used for significance testing, using $P=.05$.

\section{Results}

3.1. Formation of QD/SR9 Noncovalent Binding. To test whether SR9 peptide stably associated with QDs, QDs were mixed with SR9 at various molecular ratios $(1: 10$, $1: 20,1: 30$, and $1: 60)$. These mixtures were separated by electrophoresis in a $0.6 \%$ agarose gel (Figure 1). QD mobility decreased as the amount of SR9 increased. This indicated the formation of noncovalent QD/SR9 complexes.

3.2. Molecular Ratio of QD and SR9 Affects QD Uptake. To determine the optimal ratio for cellular uptake, A549 cells were incubated with QDs (150 nM) preincubated with SR9 


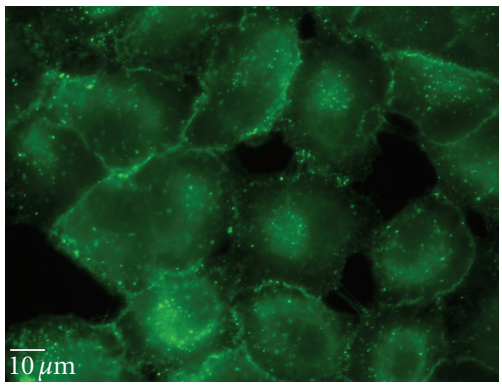

(a)

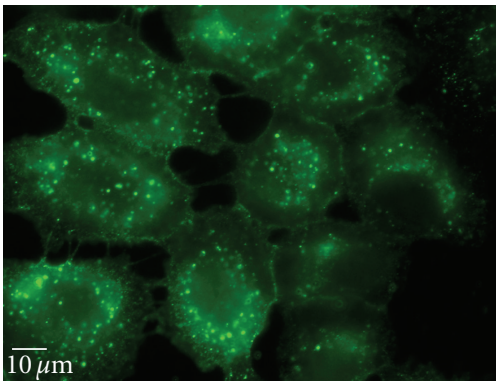

(b)

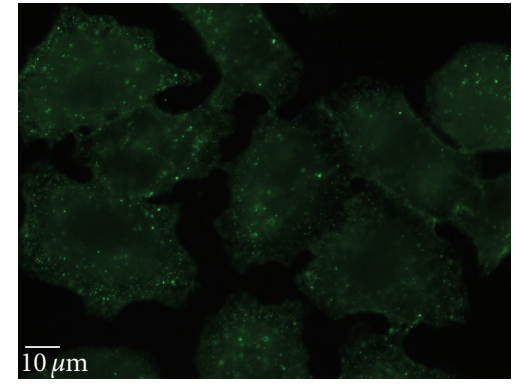

(c)

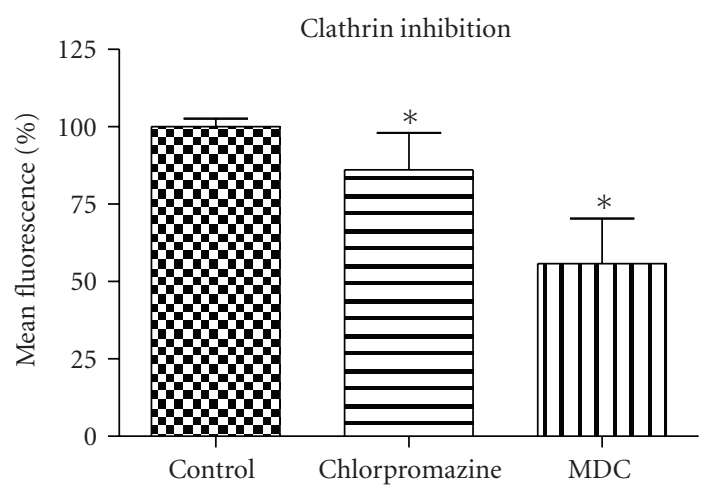

(d)

FIGURE 7: Effect of clathrin inhibitors on QD/SR9 internalization (150 nM; 1:20; 1 hour). (a) Control cells. (b) Cells pretreated with chlorpromazine for $30 \mathrm{~min}$ then incubated with QD/SR9 for an additional 1 hour. (c) Cells pretreated with MDC for 30 min then incubated with QD/SR9 for an additional 1 hour. Images are from a single plane of Z series. (d) Fluorescence intensity. Values are mean \pm SD from three independent experiments $\left({ }^{*} P^{\prime} \mathrm{s}<.01\right.$ versus control).

at different molecular ratios. QD uptake was increased as the ratio of SR9 increased from $1: 10$ to $1: 30\left(P^{\prime} s<.01\right)$ (Figure 2). However, there was no increase in the amount of internalization at ratios above $1: 30$. Although the uptake reaches the highest level at $1: 30$, good imaging quality can be obtained at $1: 20$. Further, in the future we intend to use this system to carry cargoes which requires binding sites on quantum dots. Accordingly, we chose to use $1: 20$ as the ratio between QDs and SR9.

3.3. Time-Dependent Uptake. To determine the optimal dosing time, A549 cells were incubated with QD/SR9 (1:20) for $5 \mathrm{~min}, 20 \mathrm{~min}, 40 \mathrm{~min}, 1$ hour, and 3 hours. Compared to the 5-minute group, the uptake of QD/SR9 was increased by $1.67 \pm 0.37$-fold in $20 \min \left(P^{\prime} s<.01\right)$ (Figure 3). The uptake was near maximal at 1 -hour. Accordingly, the 1 hour uptake time point was used in subsequent experiments.

3.4. Cytotoxicity of QD/SR9. To assess the cytotoxicity of QD/SR9 complex, A549 cells were exposed to QD/SR9 $(1: 20)$ complex at various QD concentrations for 24 hours. No cytotoxicity of QD/SR9 was observed at concentrations up to $200 \mathrm{nM}$ (Figure 4).

3.5. Cellular Uptake of QDs Facilitated by SR9 Peptide. To determine the ability of SR9 peptide to facilitate QD delivery, A549 cells were treated with $150 \mathrm{nM}$ QDs alone or $150 \mathrm{nM}$
QDs premixed with SR9 at a ratio of $1: 20$. After incubation of $5 \mathrm{~min}, 20 \mathrm{~min}, 40 \mathrm{~min}$, and $60 \mathrm{~min}$, QDs-treated cells were observed under an epifluorescence microscope. Images taken using the same exposure time show that enhanced uptake of the QD/SR9 complex was observed compared to QD alone (Figures 5(b)-5(e)). Quantitation of fluorescence intensities shows that compared with QD alone in five minutes, the uptake of QD/SR9 increased by 3-8-folds (Figure 5(a)).

3.6. Mechanism of $Q D / S R 9$ Internalization. Endocytosis, an essential cellular process for internalizing extracellular materials, utilizes several distinct pathways. To determine the mechanism of SR9-mediated QD delivery, a series of inhibition studies were carried out.

3.6.1. Energy-Dependent Studies. To determine whether uptake of QD/SR9 is mediated by energy-dependent endocytosis, we used low temperature or a mixture of metabolic inhibitors to deplete intracellular energy (Figure 6). In the low temperature study, the cells were incubated with QD/SR9 at $37^{\circ} \mathrm{C}$ or $4^{\circ} \mathrm{C}$. As shown in Figures 6(a) and 6(b), QD/SR9 internalization was significantly impeded at $4^{\circ} \mathrm{C}$; the fluorescence intensity at $4^{\circ} \mathrm{C}$ was $73 \pm 11.5 \%$ lower than that at $37^{\circ} \mathrm{C}$ $(P<.01)$ (Figure 6(d)). In the metabolic inhibition study, A549 cells were incubated with QD/SR9 in the presence or absence of a mixture of metabolic inhibitors $(0.15 \%$ sodium azide, $15 \mathrm{mM}$ sodium fluoride, and $2 \mu \mathrm{g} / \mathrm{mL}$ antimycin A). 


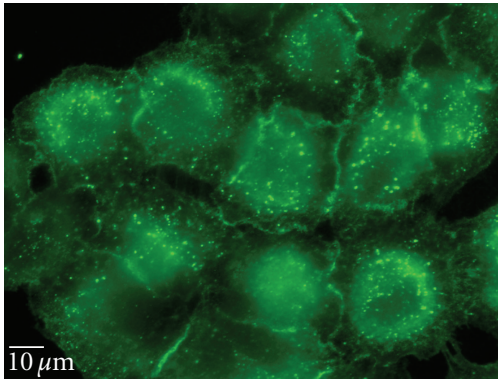

(a)

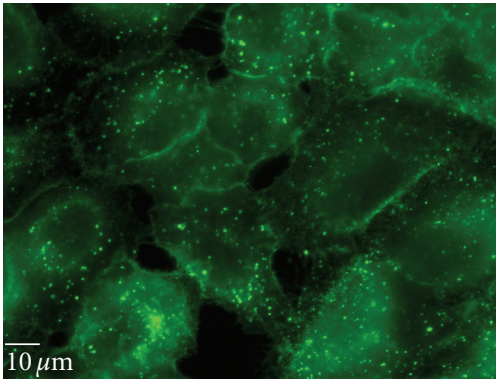

(b)

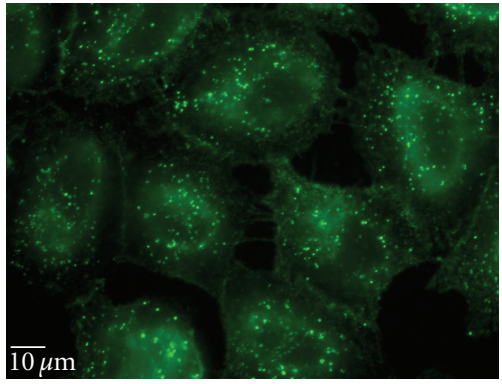

(c)

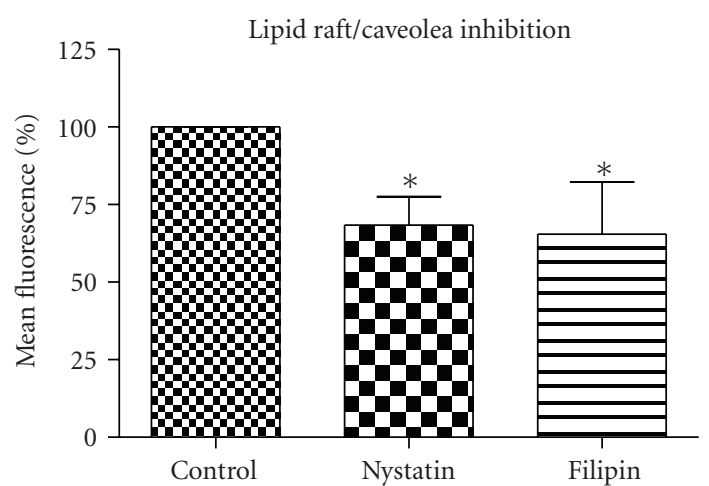

(d)

FIGURE 8: Lipid raft/caveolin inhibition decreased QD/SR9 internalization (150 nM; $1: 20$; 1 hour). (a) Control cells. (b) Cells pretreated with nystatin for $30 \mathrm{~min}$ then incubated with QD/SR9 for an additional 1 hour. (c) Cells pretreated with filipin for 30 min then incubated with QD/SR9 for an additional 1 hour. Images are from a single plane of Z series. (d) Fluorescence intensity. Values are mean \pm SD from three independent experiments $\left({ }^{*} P^{\prime} \mathrm{s}<.01\right.$ versus control).

These metabolic inhibitors strongly inhibited the uptake of QD/SR9 (Figures 6(a) and 6(c)). The fluorescent intensity in the presence of metabolic inhibitors was $61 \pm 5.7 \%$ lower than that in the control group $(P<.01)$ (Figure $6(\mathrm{~d}))$.

3.6.2. Clathrin-Dependent Pathway. To perturb the clathindependent uptake pathway, three methods were used: monodansylcadaverine (MDC), hypertonic sucrose, and chlorpromazine. The inhibitory activity of MDC is attributed to the stabilization of nascent clathrin-coated vesicles, which limits new clathrin-coated vesicle production [20]. The underlying mechanism of hypertonic sucrose involves the dispersion of clathrin lattices on the plasma membrane [21]. Chlorpromazine is a cationic amphipathic drug that relocates clathrin and adaptor protein complex-2 (AP-2) from the plasma membrane to the endosomal membrane [22]. MDC reduced the uptake of QD/SR9 by $44 \pm 13.0 \%$ $(P<.01)$ while hypertonic medium decreased uptake by $59 \%$ $(P<.01$; data not shown). In contrast, chlorpromazine only slightly reduced uptake by $14 \pm 12.1 \%(P>.05)$ (Figure 7$)$.

3.6.3. Lipid Raft/Caveolin-Dependent Pathway. Nystatin and filipin, lipid raft inhibitors, are used to block the caveolindependent endocytic pathway $[23,24]$. Treatment of cells with these two inhibitors reduced the QD/SR9 internalization by $32 \pm 8.6 \%$ and $35 \pm 15.5 \%$, respectively, $\left(P^{\prime} s<.01\right)$ (Figure 8 ), indicating that a lipid raft-dependent process is involved in QD/SR9 internalization.
3.6.4. Macropinocytosis Pathway. 5-(N-ethyl-N-isopropyl) amiloride (EIPA) is an inhibitor of the $\mathrm{Na}^{+} / \mathrm{H}^{+}$exchange that is required for macropinocytosis [25]. Cytochalasin D (Cyt D) is an F-actin depolymerizing drug that caps the barbed, faster-growing ends of actin filaments [26]. EIPA reduced the uptake of QD/SR9 by $47 \pm 14.3 \%(P<.01)$ (Figure 9). To confirm the effective concentration of Cyt D, we used Alexa 568-phalloidin to stain actin of Cyt D-treated and control cells. Actin was depolymerized in A549cells by $1 \mu \mathrm{g} / \mathrm{mL}$ Cyt D (data not shown). Cyt D significantly reduced the uptake of QDs $(P<.01)$ (Figure 9). Condensed green fluorescent agglomerates were observed around the cellular surface following treatment with Cyt D (Figure 9(c)).

3.7. siRNA Knockdown. To complement the use of pharmacological inhibitors, we used the RNAi techniques to eliminate expression of specific components of the various uptake mechanisms. Clathrin $\mathrm{HC}$ and caveolin-1 siRNA were used to knock down clathrin-dependent and caveolindepedent pathways, respectively. The Western blot experiments demonstrated the results of the knockdown treatment (Figure 10). The protein expression of clathrin and caveolin1 was reduced by $93 \pm 7.2 \%$ and $73 \pm 5.7 \%$, respectively, $\left(P^{\prime} s<.01\right)$. Following clathrin $\mathrm{HC}$ or caveolin-1 protein knockdown, the uptake of QD/SR9 was not significantly affected (Figure 11). 


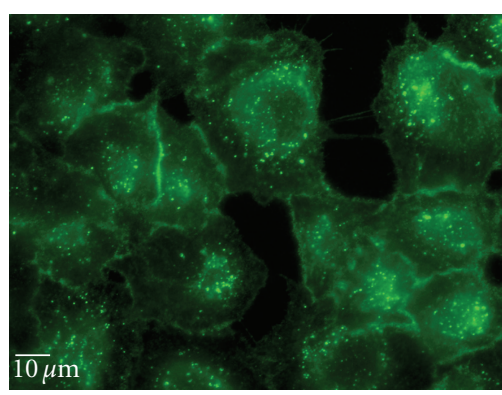

(a)

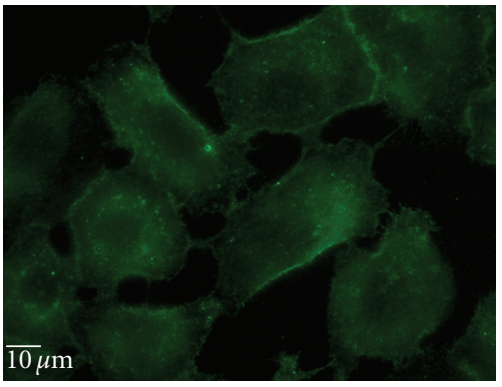

(b)

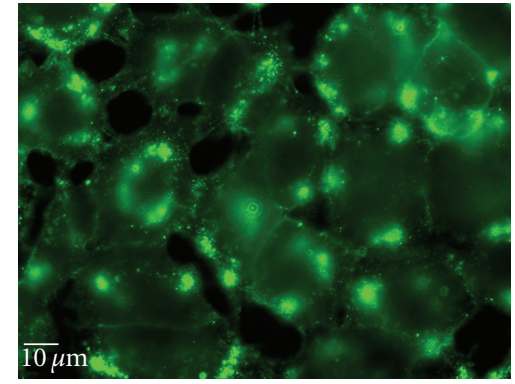

(c)

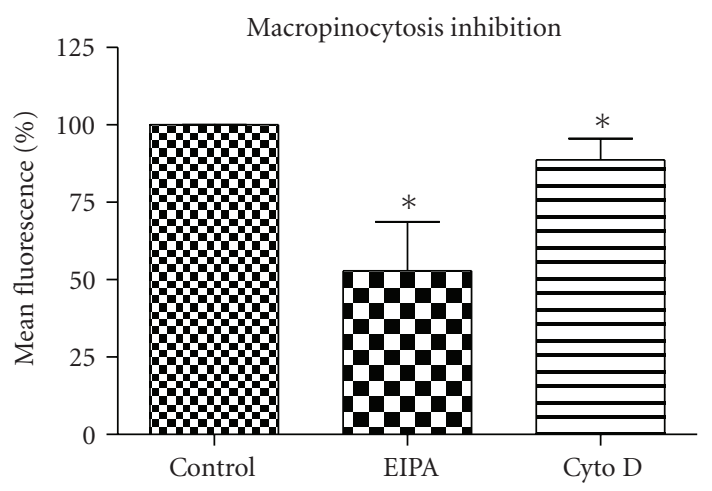

(d)

FIGURE 9: Macropinocytosis inhibition perturbed QD/SR9 internalization (150 nM; $1: 20 ; 1$ hour). (a) Control cells. (b) Cells pretreated with EIPA for 30 min then incubated with QD/SR9 for an additional 1 hour. (c) Cells pretreated with Cyt D for 30 min then incubated with QD/SR9 for an additional 1 hour. Images are from a single plane of Z series. (d) Fluorescence intensity. Values are mean \pm SD from three independent experiments $\left({ }^{*} P^{\prime}\right.$ s $<.01$ versus control).

\section{Discussion}

Quantum dots are an emerging class of fluorescent probes for cellular imaging. In comparison with organic dyes and fluorescent proteins, QDs have unique properties, including high quantum yield and photostability, which make them better suited for delivering and monitoring biomolecules. The efficiency of internalizing QDs can be improved by incorporating cell-penetrating peptides [5]. Accordingly, our goals were to test whether nona-arginine (SR9) facilitates QD uptake and to determine the mechanism of this uptake. Data from this study demonstrate that QDs are internalized into A549 cells within 5 min with the aid of SR9; without SR9, QD uptake is minimal (Figure 5). The enhancement of internalization by basic protein transducers is thought to reflect an electrostatic interaction between cationic nonaarginine and the negatively charged polar heads of the phospholipids of the plasma membrane [18]. Our study demonstrates that SR9 can significantly increase QD uptake.

QD/SR9 internalization was dramatically blocked by either low temperature or a mixture of metabolic inhibitors. This indicates that the uptake of QD/SR9 is highly energydependent and supports the notion that endocytosis is the major uptake process. Since endocytosis encompasses numerous pathways, our subsequent studies were designed to further elucidate the specific mechanism of uptake.
Clathrin-dependent endocytosis is a well-characterized endocytic pathway. To determine the role of clathrinmediated uptake of QD/SR9, we used three different inhibitors. Treating cells with hypertonic sucrose significantly reduced QD/SR9 uptake. Although this kind of inhibition is considered evidence of clathrin-dependent endocytosis, recent studies have suggested that it also interferes with other internalization pathways, including caveolin-dependent endocytosis and macropinocytosis [27]. Accordingly, the effects of two additional inhibitors, MDC and chlorpromazine, were determined. MDC significantly impeded QD/SR9 internalization. Though MDC is considered as a clathrin pathway inhibitor, it is also a macropinocytosis inhibitor via suppression of the transglutaminase family that activates Rho GTPases, the key regulators of actin assembly and dynamics [27]. Thus, MDC might block actindependent pathways such as macropinocytosis. Chlorpromazine had only a small effect on uptake. Due to the varying results and possible off-target effects of the inhibitor studies, we used siRNA to knock down clathrin heavy chain. The Western blot analysis indicated that near complete clathrin protein $(93 \pm 5.7 \%)$ expression was suppressed. The almost complete loss of clathrin did not affect the QD fluorescence intensity inside cells relative to control levels. Together, our data suggest that clathrin-mediated endocytosis is not required for QD/SR9 internalization. However, because 


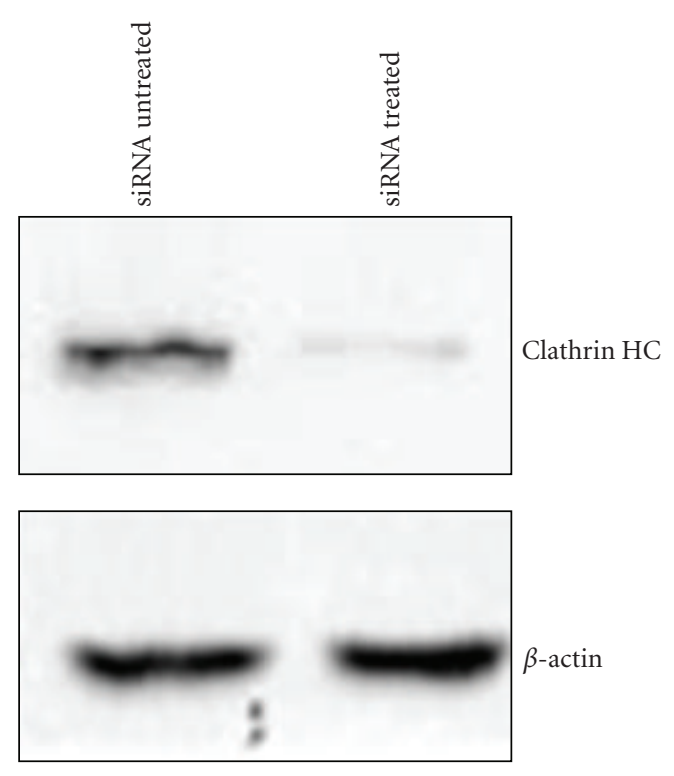

(a)
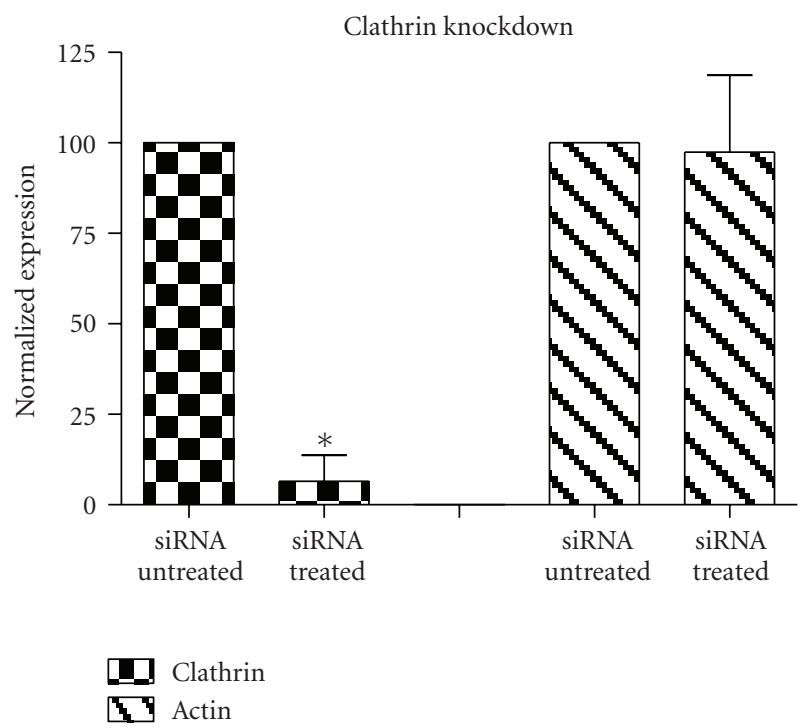

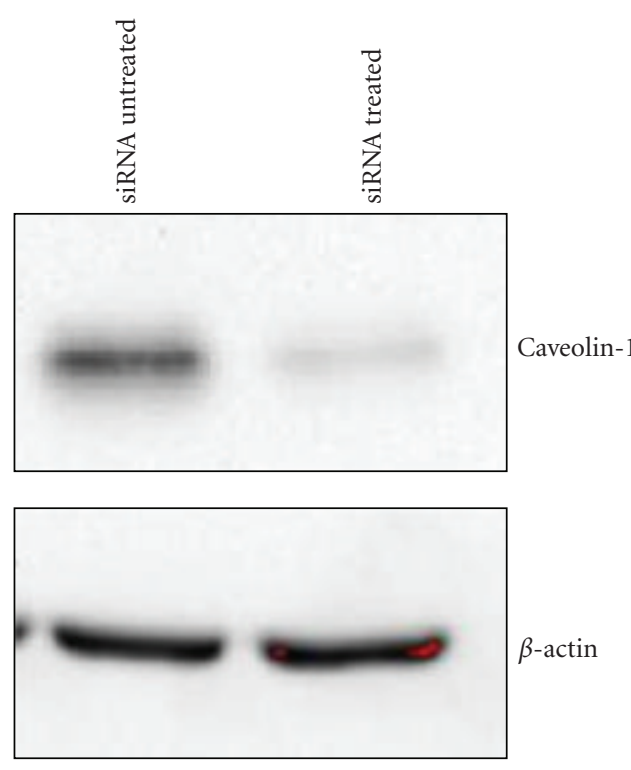

(b)

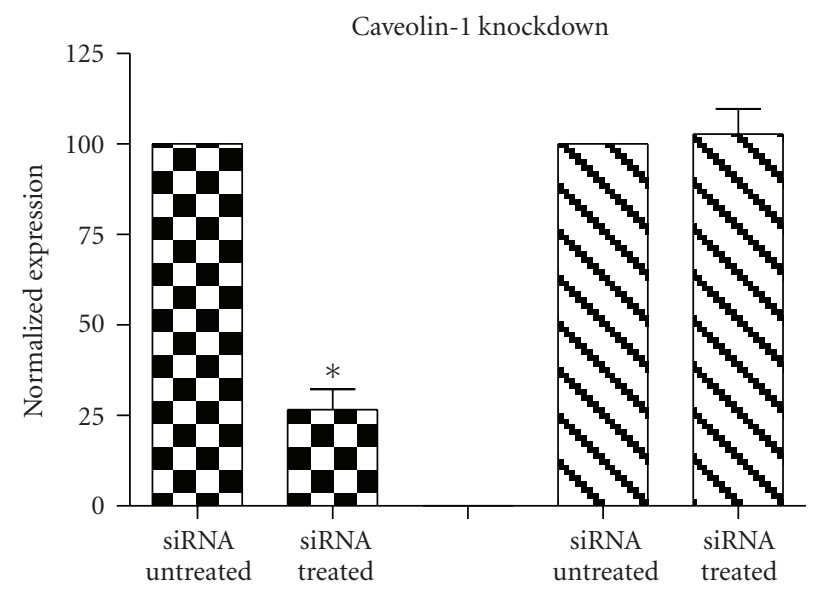

므 Caveolin-1 Actin

(c)

(d)

FIgURE 10: Western blot analysis of clathrin HC and caveolin-1 protein after siRNA treatment. A549 cells were treated with siRNA of clathrin HC or caveolin-1 protein for 3 days, and then cells were lysed for Western blot analysis. (a) Clathrin HC protein expression in A549 cells. (b) Caveolin-1 protein expression in A549 cells. $\beta$-actin was used as the internal control. (c) and (d) Quantification analysis of the Western blots. Intensity is normalized to the siRNA untreated group. Values are mean \pm SD from three independent experiments $\left({ }^{*} P^{\prime}\right.$ s $<.01$ versus control).

clathrin protein was not completely eliminated, we cannot rule out that clathrin is involved in QD/SR9 uptake.

Nystatin and filipin are polyene antibiotics that accumulate cholesterol and thereby sequester lipid from cell membranes. Nystatin and filipin are highly selective inhibitors of the lipid raft-dependent pathway because of this lipid sequestering $[23,24]$. Both inhibitors hindered the uptake of QD/SR9, suggesting that the internalization was lipid raft dependent. Cholesterol depletion could result in blockage of several lipid raft-dependent endocytic pathways, including caveolae-dependent and macropinocytic pathways [24]. For further clarification, caveolin-1 siRNA was used to knockdown caveolin-1 expression. The Western blot analysis and live cell imaging indicated that internalization of QD/SR9 was not reduced while caveolin-1 protein was knocked down by $73 \pm 7.2 \%$. These findings suggest that QD/SR9 internalization is a lipid raft-dependent process that is not mainly caveolin-mediated. Because caveolin-1 protein was not completely knock down, we cannot rule out that caveolin-1 was involved in QD/SR9 uptake. 


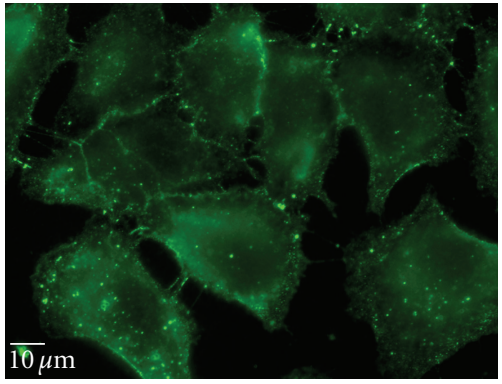

(a)

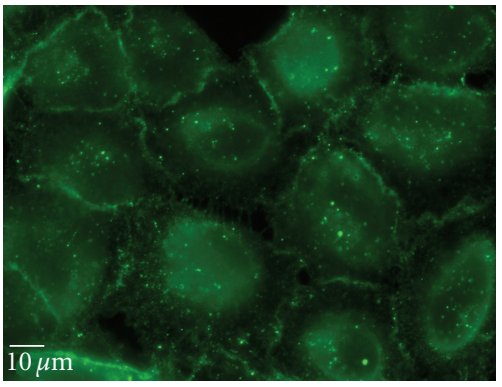

(b)

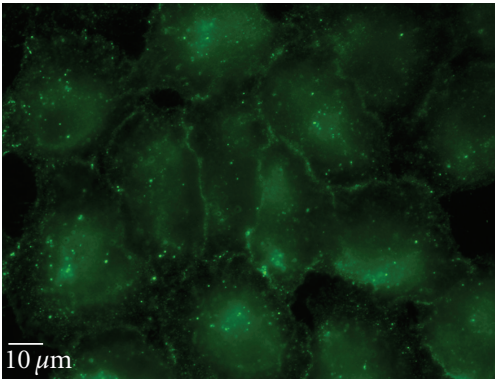

(c)

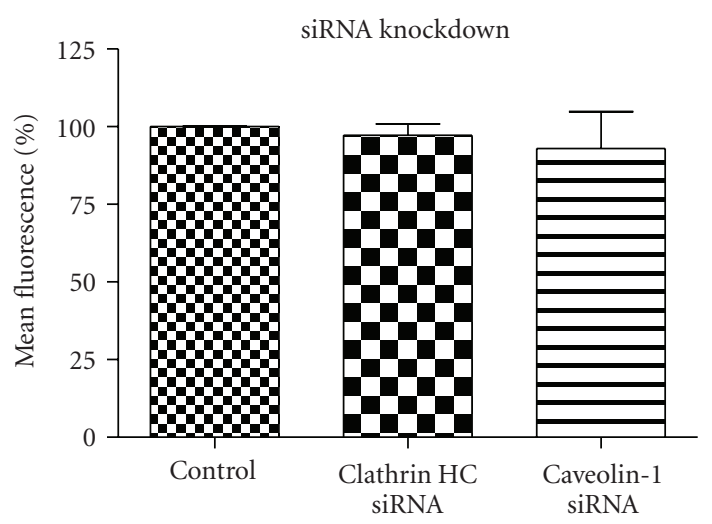

(d)

FIGURE 11: QD/SR9 uptake after knockdown of clathrin HC and caveolin-1 protein (150 nM; $1: 20 ; 1$ hour). A549 cells were treated with siRNA of clathrin HC or caveolin-1 protein for 3 days, and then cells were used for uptake studies. (a) Control cells. (b) Cells pretreated with clathrin HC siRNA. (c) Cells pretreated with caveolin-1 siRNA. Images are from a single plane of Z series. (d) Fluorescence intensity. Values are mean \pm SD from three independent experiments.

Macropinocytosis is a rapid, lipid raft-dependent, and receptor-independent form of endocytosis. Macropinocytosis includes three essential steps: actin cytoskeleton-driven ruffle formation, closure of the ruffle into a vesicle, and dissociation of actin filaments from the vesicle [28]. The fluorescent intensities in cells treated with two macropinocytosis inhibitors, EIPA and Cyt D, were significantly reduced compared to the control cells. It is worth noting that in Cyt D-treated cells, QD/SR9 was densely localized around cellular surfaces, not in cytosol, leading to overestimate of QD/SR9 internalization. This is similar with the observation of Nakase et al. that cellular R8 peptide was predominantly localized in cellular boundaries with very little punctuate staining in Cyt D-treated cells [29]. Ruan et al. made a similar observation with Cyt D-treated cells [6]. It is unclear how the dense aggregates affect quantitation. In summary the uptake of QD/SR9 is lipid raft-dependent macropinocytosis.

Collectively, our data show that SR9 facilitates a rapid energy-dependent cellular entry of QDs. The internalization involves lipid raft and depends on macropinocytosis. Other pathways such as RhoA-dependent and CDC42-dependent endocytic pathways may also deserve attention [30,31].

\section{Acknowledgments}

The authors thank Robert S. Aronstam for technical editing and Chuan-Chin Huang and Hsiu Jen Wang for technical support. This work was supported by Award No. R15EB009530 from the National Institute of Biomedical Imaging and Bioengineering (to Y.-w. Huang) and the National Science Council (NSC 97-2621-B-259-003-MY3 to H.-J. Lee), Taiwan.

\section{References}

[1] X. Michalet, F. F. Pinaud, L. A. Bentolila et al., "Quantum dots for live cells, in vivo imaging, and diagnostics," Science, vol. 307, no. 5709, pp. 538-544, 2005.

[2] D. J. Bharali, D. W. Lucey, H. Jayakumar, H. E. Pudavar, and P. N. Prasad, "Folate-receptor-mediated delivery of InP quantum dots for bioimaging using confocal and two-photon microscopy," Journal of the American Chemical Society, vol. 127, no. 32, pp. 11364-11371, 2005.

[3] A. Hoshino, K. Fujioka, T. Oku et al., "Quantum dots targeted to the assigned organelle in living cells," Microbiology and Immunology, vol. 48, no. 12, pp. 985-994, 2004.

[4] F. Chen and D. Gerion, "Fluorescent CdSe/ZnS nanocrystalpeptide conjugates for long-term, nontoxic imaging and nuclear targeting in living cells," Nano Letters, vol. 4, no. 10, pp. 1827-1832, 2004.

[5] F. L. Xue, J. Y. Chen, J. Guo et al., "Enhancement of intracellular delivery of CdTe quantum dots (QDs) to living cells by tat conjugation," Journal of Fluorescence, vol. 17, no. 2, pp. 149-154, 2007.

[6] G. Ruan, A. Agrawal, A. I. Marcus, and S. Nie, "Imaging and tracking of Tat peptide-conjugated quantum dots in 
living cells: new insights into nanoparticle uptake, intracellular transport, and vesicle shedding," Journal of the American Chemical Society, vol. 129, no. 47, pp. 14759-14766, 2007.

[7] L. W. Zhang and N. A. Monteiro-Riviere, "Mechanisms of quantum dot nanoparticle cellular uptake," Toxicological Sciences, vol. 110, no. 1, pp. 138-155, 2009.

[8] G. P. H. Dietz and M. Bähr, "Delivery of bioactive molecules into the cell: the Trojan horse approach," Molecular and Cellular Neuroscience, vol. 27, no. 2, pp. 85-131, 2004.

[9] Y.-H. Wang, Y.-W. Hou, and H.-J. Lee, "An intracellular delivery method for siRNA by an arginine-rich peptide," Journal of Biochemical and Biophysical Methods, vol. 70, no. 4, pp. 579-586, 2007.

[10] G. Tünnemann, G. Ter-Avetisyan, R. M. Martin, M. Stöckl, A. Herrmann, and M. C. Cardoso, "Live-cell analysis of cell penetration ability and toxicity of oligo-arginines," Journal of Peptide Science, vol. 14, no. 4, pp. 469-476, 2008.

[11] A. Ziegler, P. Nervi, M. Dürrenberger, and J. Seelig, "The cationic cell-penetrating peptide CPPTAT derived from the HIV-1 protein TAT is rapidly transported into living fibroblasts: optical, biophysical, and metabolic evidence," Biochemistry, vol. 44, no. 1, pp. 138-148, 2005.

[12] S. Futaki, "Arginine-rich peptides: potential for intracellular delivery of macromolecules and the mystery of the translocation mechanisms," International Journal of Pharmaceutics, vol. 245, no. 1-2, pp. 1-7, 2002.

[13] Y.-H. Wang, C.-P. Chen, M.-H. Chan et al., "Argininerich intracellular delivery peptides noncovalently transport protein into living cells," Biochemical and Biophysical Research Communications, vol. 346, no. 3, pp. 758-767, 2006.

[14] M. A. Lindsay, "Peptide-mediated cell delivery: application in protein target validation," Current Opinion in Pharmacology, vol. 2, no. 5, pp. 587-594, 2002.

[15] S. R. Schwarze, K. A. Hruska, and S. F. Dowdy, "Protein transduction: unrestricted delivery into all cells?" Trends in Cell Biology, vol. 10, no. 7, pp. 290-295, 2000.

[16] J. S. Wadia and S. F. Dowdy, "Protein transduction technology," Current Opinion in Biotechnology, vol. 13, no. 1, pp. 5256, 2002.

[17] L. N. Patel, J. L. Zaro, and W.-C. Shen, "Cell penetrating peptides: intracellular pathways and pharmaceutical perspectives," Pharmaceutical Research, vol. 24, no. 11, pp. 1977-1992, 2007.

[18] E. L. Snyder and S. F. Dowdy, "Cell penetrating peptides in drug delivery," Pharmaceutical Research, vol. 21, no. 3, pp. 389-393, 2004.

[19] M. Chang, J.-C. Chou, C.-P. Chen, B. R. Liu, and H.-J. Lee, "Noncovalent protein transduction in plant cells by macropinocytosis," New Phytologist, vol. 174, no. 1, pp. 46-56, 2007.

[20] Y. Phonphok and K. S. Rosenthal, "Stabilization of clathrin coated vesicles by amantadine, tromantadine and other hydrophobic amines," FEBS Letters, vol. 281, no. 1-2, pp. 188190, 1991.

[21] S. H. Hansen, K. Sandvig, and B. van Deurs, "Clathrin and HA2 adaptors: effects of potassium depletion, hypertonic medium, and cytosol acidification," Journal of Cell Biology, vol. 121, no. 1, pp. 61-72, 1993.

[22] L.-H. Wang, K. G. Rothberg, and R. G. W. Anderson, "Misassembly of clathrin lattices on endosomes reveals a regulatory switch for coated pit formation," Journal of Cell Biology, vol. 123, no. 5, pp. 1107-1117, 1993.

[23] I. A. Khalil, K. Kogure, S. Futaki, and H. Harashima, "High density of octaarginine stimulates macropinocytosis leading to efficient intracellular trafficking for gene expression," Journal of Biological Chemistry, vol. 281, no. 6, pp. 3544-3551, 2006.

[24] J. S. Wadia, R. V. Stan, and S. F. Dowdy, "Transducible TATHA fusogenic peptide enhances escape of TAT-fusion proteins after lipid raft macropinocytosis," Nature Medicine, vol. 10, no. 3, pp. 310-315, 2004.

[25] L. J. Hewlett, A. R. Prescott, and C. Watts, "The coated pit and macropinocytic pathways serve distinct endosome populations," Journal of Cell Biology, vol. 124, no. 5, pp. 689703, 1994.

[26] J. A. Cooper, "Effects of cytochalasin and phalloidin on actin," Journal of Cell Biology, vol. 105, no. 4, pp. 1473-1478, 1987.

[27] A. I. Ivanov, Exocytosis and Endocytosis, Humana Press, Totowa, NJ, USA, 2007.

[28] J. A. Swanson and C. Watts, "Macropinocytosis," Trends in Cell Biology, vol. 5, no. 11, pp. 424-428, 1995.

[29] I. Nakase, M. Niwa, T. Takeuchi et al., "Cellular uptake of arginine-rich peptides: roles for macropinocytosis and actin rearrangement," Molecular Therapy, vol. 10, no. 6, pp. 10111022, 2004.

[30] S. Mayor and R. E. Pagano, "Pathways of clathrin-independent endocytosis," Nature Reviews Molecular Cell Biology, vol. 8, no. 8, pp. 603-612, 2007.

[31] K. Sandvig, M. L. Torgersen, H. A. Raa, and B. Van Deurs, "Clathrin-independent endocytosis: from nonexisting to an extreme degree of complexity," Histochemistry and Cell Biology, vol. 129, no. 3, pp. 267-276, 2008. 

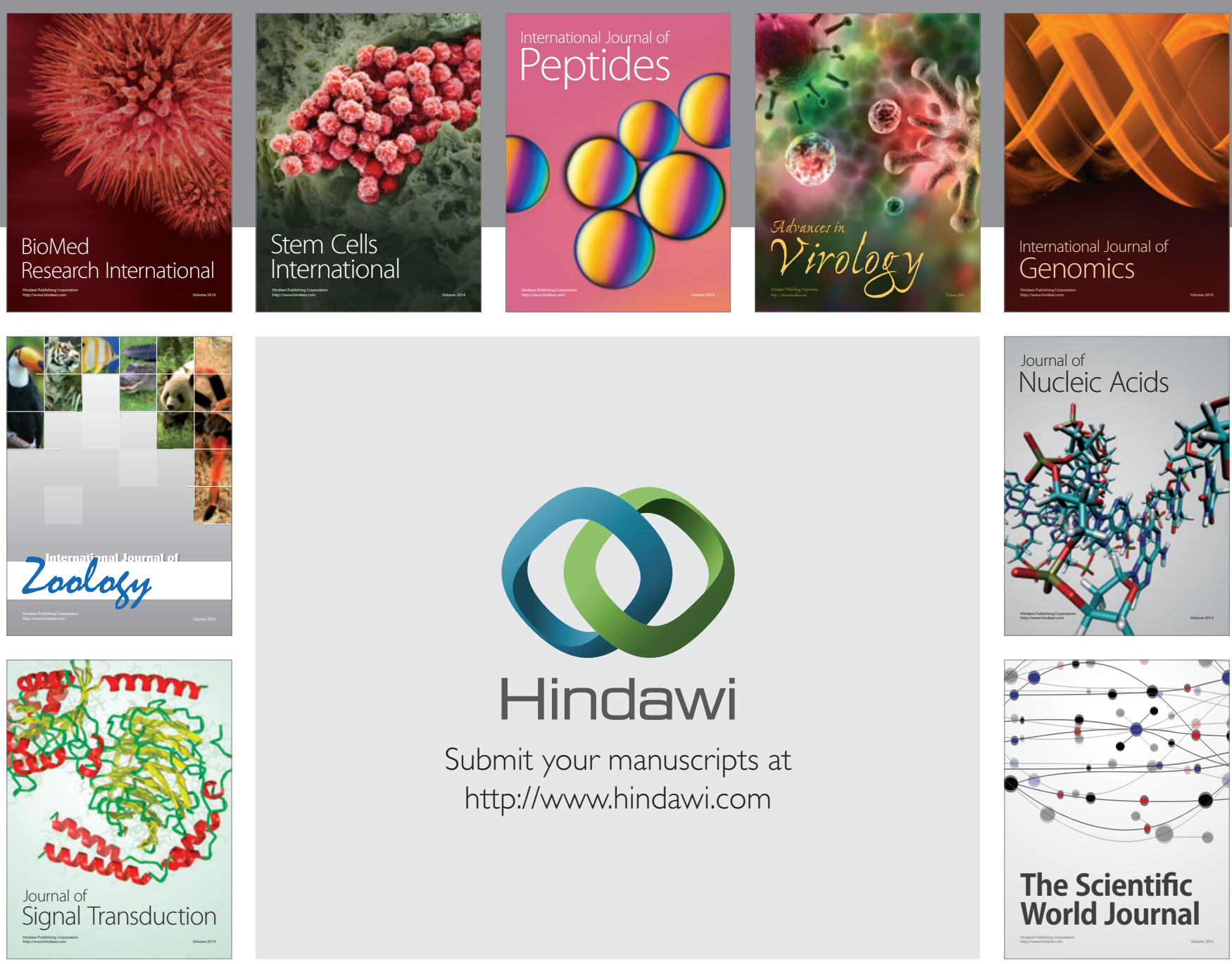

Submit your manuscripts at

http://www.hindawi.com
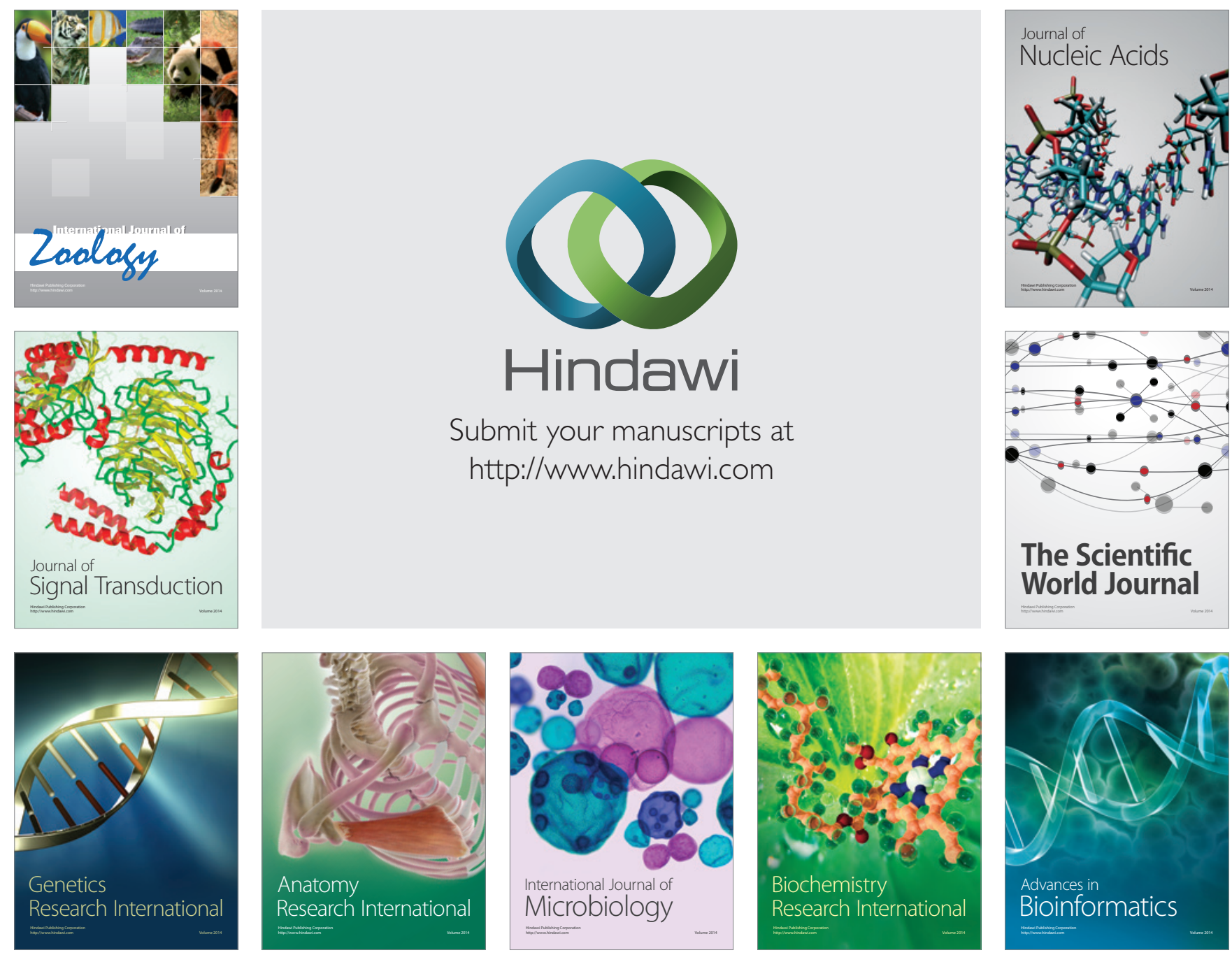

The Scientific World Journal
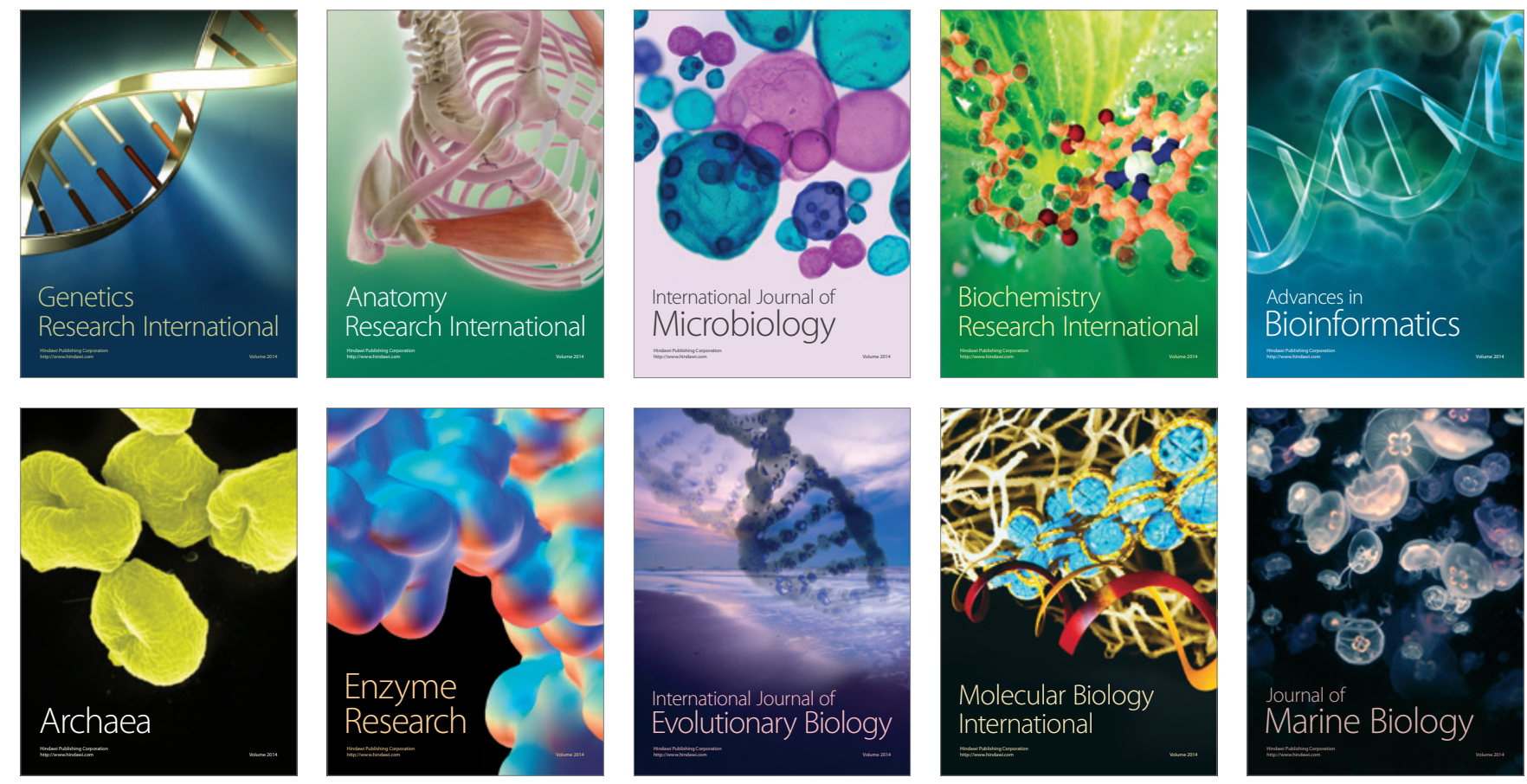